\title{
SELEÇÃO DE ÁRVORES MATRIZES DA ESPÉCIE Astronium concinnum SCHOTT EM DOIS FRAGMENTOS FLORESTAIS DO SUL DO ESPIRITO SANTO
}

\author{
Alessandra Abreu Rodrigues Vieira ${ }^{1}$ \\ Aléxia Gonçalves Pereira ${ }^{2}$ \\ Adelson Lemes da Silva Júnior ${ }^{3}$ \\ Lucimara Cruz de Souza ${ }^{4}$ \\ Mariana Cruz de Souza ${ }^{5}$ \\ Kelmer Mozer Moro 6 \\ Marcos Vinicius Winckler Caldeira ${ }^{7}$ \\ Fábio Demolinari de Miranda ${ }^{8}$
}

Resumo: A Floresta Atlântica é considerada um dos ecossistemas mais ricos em biodiversidade, por outro lado, a intensa extração de material vegetal devido aos sucessivos ciclos econômicos, reduziu esse sistema transformando-o em um grande conjunto de fragmentos. A seleção de árvores matrizes para coleta de sementes vem se destacando como ferramenta no manejo e ampliação da cobertura vegetal. Dessa forma, objetivou-se com o presente estudo analisar o potencial de árvores de Astronium concinnum Schott, presentes em duas unidades de conservação inseridas no município de Cachoeiro de Itapemirim, Espírito Santo, como matrizes fornecedoras de sementes. Foram identificadas e selecionadas 48 árvores adultas de $A$. concinnum que se apresentaram com boas condições de sanidade. O maior valor mensurado para $O$ diâmetro a altura do peito (DAP) foi de $47,9 \mathrm{~cm}$ e o menor DAP foi de $5 \mathrm{~cm}$, com uma média de $16,68 \mathrm{~cm}$. Os dados fornecidos com esse estudo servirão de base para projetos de reflorestamento, uma vez que possibilitou a identificação de árvores matrizes fornecedoras de semente.

Palavras-chave: Preservação; Remanescentes florestais; Reflorestamento; Sementes.

\footnotetext{
1 Universidade Federal do Espírito Santo - Centro de Ciências Agrárias / Programa de Pós-Graduação em Genética e Melhoramento. E-mail: abreuepires@gmail.com.

2 Universidade Federal do Espírito Santo - Centro de Ciências Agrárias / Programa de Pós-Graduação em Genética e Melhoramento. E-mail: alexiagp@gmail.com.

3 Universidade Federal do Espírito Santo - Centro de Ciências Agrárias / Programa de Pós-Graduação em Genética e Melhoramento. E-mail: adelsonlemes@yahoo.com.br.

4 Universidade Federal do Espírito Santo - Centro de Ciências Agrárias / Programa de Pós-Graduação em Genética e Melhoramento. E-mail: lucimaracruz15@hotmail.com.

5 Universidade Federal do Espírito Santo - Centro de Ciências Agrárias / Programa de Pós-Graduação em Genética e Melhoramento. E-mail: scruz.mariana@gmail.com.

6 Universidade Federal do Espírito Santo - Centro de Ciências Agrárias / Programa de Pós-Graduação em Genética e Melhoramento. E-mail: kelmer.moro@gmail.com.

7 Universidade Federal do Espírito Santo - Centro de Ciências Agrárias / Programa de Pós-Graduação em Genética e Melhoramento. E-mail: mvwcaldeira@gmail.com.

8 Universidade Federal do Espírito Santo - Centro de Ciências Agrárias / Programa de Pós-Graduação em Genética e Melhoramento. E-mail: fademolinari@yahoo.com.br.
} 